\title{
La vinculación universitaria con las empresas
}

\section{MARTÍN EDUARDO PÉREZ-CAZARES ${ }^{a}$}

\begin{abstract}
RESUMEN La falta de producción científica en las universidades por parte de sus catedráticos, y una escasa vinculación entre estudiante y maestro, produce una pobre motivación en el alumnado para la investigación de temas que tiendan a un mejor desarrollo en la producción de las empresas. Esto, aunado a la escasa infraestructura en bibliotecas y la falta de apoyo económico para tener acceso a revistas de otras universidades, tanto nacionales, como extranjeras, así como revistas científicas que sirvan no solo de apoyo al estudiante, sino de aliento para generar nuevas ideas, conlleva en gran parte al déficit en la investigación científica en México, a fin de de generar ciencia. En una comparativa latinoamericana, países como Argentina, Chile y Brasil tienen mayores niveles de investigación con relación con el país azteca. En la presente investigación se busca encontrar ideas nuevas que se tomen en cuenta en el propósito de robustecer la insuficiente investigación de este país.
\end{abstract}

PALABRAS CLAVE empresa, ciencia, educación, investigación, método.

\section{HISTORIA DEL ARTÍCULO}

¿CÓMO CITAR?:

Pérez-Cazares, M. E. (2017). La vinculación universitaria con las empresas. Perspectiva Empresarial, 4(2), 37-46. http://dx.doi. org/10.16967/rpe.v4n2a4

RECIBIDO: 30 de julio de 2017 APROBADO: 25 de agosto de 2017

CORRESPONDENCIA:

Martín Eduardo Pérez Cazares, Universidad de Guadalajara, Av. Juárez No. 976, Colonia Centro. C.P. 44100, Guadalajara, México.

a Doctor en derecho y Doctor en ciencias políticas y sociales, profesor investigador de tiempo completo en la Universidad de Guadalajara.Correo electrónico:mperezcazares@yahoo.com.mx 


\section{¿CÓMO CITO EL ARTÍCULO? HOW TO CITE THIS PAPER?}

CHICAGO:

Pérez-Cazares, Martín

Eduardo. 2017. “La vinculación universitaria con las empresas". Perspectiva

Empresarial 4(2): 37-46. http://dx.doi.org/10.16967/ rpe.v4n2a4

MLA:

Pérez-Cazares, Martín Eduardo. "La vinculación universitaria con las empresas". Perspectiva Empresarial 4.2 (2017): 37-46. Digital. http://dx.doi. org/10.16967/rpe.v4n2a4

\section{The university-company relationship}

ABSTRACT The lack of scientific production at universities by their professors and a poor connection between student and teacher result in the student body's poor motivation to do research that tends to improve production at companies. This, coupled with the scarce library infrastructure and the absence of economic support to have access to journals from other universities, both national and foreign, and scientific journals that serve not only to support the student, but also to encourage new ideas, has led to a deficit in scientific research in Mexico. In a Latin American comparison, countries like Argentina, Chile and Brazil have higher levels of research in relation to the Aztec country. This paper seeks to find new ideas that should be considered to strengthen research and generate science in this country.

KEYWORDS company, science, education, research, method.

\section{O vínculo universitário com as empresas}

RESUMO A falta de produção científica nas universidades por parte dos seus professores, e um escasso vínculo entre estudante e docente, produz uma pobre motivação nos alunos para a pesquisa de temas que tendem a um melhor desenvolvimento na produção das empresas. Isso, somado à escassa infraestrutura nas bibliotecas e à falta de apoio econômico para ter acesso a revistas de outras universidades, tanto nacionais quanto estrangeiras, assim como revistas científicas que sirvam não só de apoio ao estudante, senão que de inspiração para gerar novas ideias, implica em grande parte no déficit da pesquisa científica no México, a fim de gerar ciência. Em uma análise comparativa latino -americana, países como a Argentina, o Chile e o Brasil têm maiores níveis de pesquisa em relação ao país asteca. Na presente pesquisa busca-se encontrar ideias novas que sejam tidas em conta com o objetivo de fortalecer a pesquisa no México, que hoje é insuficiente.

PALAVRAS CHAVE ciência, educação, empresa, método, pesquisa. 


\section{Educación, investigación y vinculación}

La vinculación se conceptualiza como la relación que se establece entre una persona y otra con intereses afines, desde generales hasta particulares, bien sea en mercaderías, o bien en compraventas, y que tienen influencia entre sí para llevar a cabo ciertos objetivos comunes (Enciclopedia Jurídica Mexicana, 2012, pp. 988-990).

La vinculación universitaria es la relación que se establece entre la universidad con los sectores productivos de bienes o servicios (públicos o privados), con el propósito de establecer intereses afines y lograr avances significativos en la transmisión del conocimiento.

En este segundo decenio del siglo XXI, en el que la libertad de empresa y la libre competencia, por medio del liberalismo, se han arraigado con más ahínco en el ánimo de los gobiernos, es necesaria una cercanía más estrecha entre las universidades y las empresas, a fin de conocer las necesidades que estas requieren y así formar a los profesionistas con el currículo adecuado a cada rama de la producción, de servicios o de bienes.

Uno de los mayores desafíos de cualquier país es impulsar la educación y la investigación, con el fin de abordar los problemas sociales de manera sistemática y, sobre todo, ofrecer alternativas de soluciones pertinentes y viables. Importante es resaltar la misión de quienes impartimos cátedra a nivel licenciatura, maestría o doctorado, en relación con formar recursos humanos para generar nuevas investigaciones que tiendan a resolver las necesidades sociales mediante nuevos conocimientos que permitan reducir costos de producción y lograr una mayor competitividad en los mercados globalizados. Esto en razón a que, día a día, se enfrentan nuevos retos y exigencias al surgir nuevas formas y costumbres sociales que bien merecen un esfuerzo riguroso en la investigación $y$, desde luego, en la enseñanza, en busca, sobre todo, de medios constructivos que tiendan a crear nuevos mecanismos de producción para mejorar la convivencia.

De acuerdo con Rodríguez, Rojas \& Lobato (2015, p.18):

La interacción universidad-empresa se ha fortalecido en muchos países, debido al incremento del apoyo gubernamental a los centros de investigación y producción de conocimientos, al decidido interés de algunas empresas privadas en establecer equipos interdisciplinarios para los procesos de investigación y desarrollo requeridos en sus áreas de negocios y al cambio de mentalidad en la gestión de las instituciones académicas por parte de sus dirigentes. Por ello hoy se vive un profundo cambio de los modelos sociales respecto a la gestión tradicional de la universidad, pasando de una visión esencialmente académica a una visión empresarial complementaria.

Por tanto, se debe ofrecer al alumno un panorama amplio de la problemática que enfrenta el investigador, con el fin de delimitar y definir el conjunto de problemas que sean susceptibles de ser investigados, el manejo y uso de los conocimientos por parte de distintos sectores sociales que representan temas a investigar para el desarrollo del conocimiento científico, así como las soluciones prácticas que pudieran darse con el apoyo, tanto del gobierno, como de la iniciativa privada.

En este sentido, la triple hélice surge como respuesta a la creciente necesidad de relacionar estrechamente la actividad científica tecnológica y productiva, con el objeto de hacer frente a la demanda del mercado (Chang, 2010, p. 86). González (2009) refiere que este modelo se centra en el análisis de las relaciones mutuas entre las universidades y los entornos científicos como primera hélice, las empresas e industrias como segunda, y las administraciones y el gobierno como tercera.

Las condiciones del mundo actual plantean el reto de saber si las ideas con las que se analizan las nuevas realidades son o no las más adecuadas o apropiadas. Desde esta perspectiva, es claro que se debe asumir que en el campo del conocimiento, y en relación con la educación universitaria, muchos de nuestros conceptos - que hasta hace poco eran útiles-, en este momento descubren su incapacidad de explicar los sucesos que en ambos terrenos aparecen. Lo anterior exige una postura radical y valiente para realizar un giro extremo y aventurar nuevas expectativas de conocimiento (Larrauri, 2006, p. 64).

Reclutar al personal académico por medio del anuncio abierto y sobre una base del mérito por parte de las empresas, así como crear un sistema fuerte de ejecución en la gerencia de funcionamiento que deba ser vinculatorio con las universidades, son medidas para mejorar prácticas de enseñanza y garantizar la calidad en la inversión y en la formación de capital humano, con base en un modelo de educación que se adapte a 
las necesidades de la empresa, y así se satisfaga la adquisición de conocimiento que el desarrollo del país requiere para una economía global competitiva.

Ante dicha competitividad, las universidades de México deben aplicar la enseñanza de métodos y técnicas como tema primordial en cada cátedra que se imparta, a fin de luego también aplicar los métodos y técnicas a los procesos de producción en las empresas. Esto en el sentido en que la universidad es el proveedor de recursos humanos en los factores de producción; si como universidad entregamos un producto de alta calidad y competencial al empresariado, esto robustecerá la calidad y la cantidad en la producción de una empresa.

Todo esto no podría dar frutos sino es con el apoyo gubernamental, al crear políticas públicas para el desarrollo del conocimiento. De ahí la importancia de una vinculación estrecha entre gobierno, universidad y empresa, ejes necesarios para la trasferencia del conocimiento.

\section{La aplicación de los métodos en la universidad}

A primera vista, cuando se habla de los métodos del conocimiento, pareciera que se hace referencia solo al conocimiento en los laboratorios, en las academias o en los institutos. Sin embargo, si nos detenemos un momento, se puede observar cómo, incluso el más ignorante de los hombres, necesariamente está aplicando todos estos métodos. Lo que acontece es que lo hace muchas veces sin ser consciente de ello.

Ahora bien, la capacidad cognoscitiva del hombre es posible mediante la utilización de los conceptos generales. Es esto lo que permite comparar y relacionar, de forma que se eviten una serie de repeticiones inútiles $\mathrm{y}$, dado el momento en que el alumno construya o elabore un concepto, no lo olvidará. En este sentido, la inducción y la deducción son métodos que se implican mutuamente, pues por medio de la experiencia el hombre empieza a formular principios generales a partir de los cuales puede saber cómo se manejan los hechos particulares posteriores. En consecuencia, el conocimiento humano se compone de una serie de principios generales que le enseñan al ser humano cómo manejar los hechos concretos. Esto, a su vez, le permite al sujeto ampliar la extensión de esos principios generales, y así sucesivamente.
Con base en lo anterior, se puede aducir que todo el conocimiento práctico y teórico es posible a través de los casos que le van sucediendo al hombre a lo largo de su historia. Incluso, el aprendizaje y la captación de los principios lógicos, matemáticos y geométricos se da a partir de ejemplos concretos. Recordemos que la ciencia y la cultura se han transmitido de generación en generación, al superar con el análisis y el estudio los anteriores conocimientos, y así llegan a formar y crear nuevos, construir nuevas teorías y realizar nuevos descubrimientos. Obsérvese cómo, desde la enseñanza primaria, los estudiantes aprenden los principios generales mediante ejercicios prácticos impuestos por el profesor. Aunque su aprehensión es posible en abstracto, lo cierto es que en la vida diaria se requiere la aplicación de un silogismo mediante el cual los problemas concretos constituyen premisas menores, y su solución se logra mediante la subsunsión de esa premisa menor dentro de la premisa mayor (concepto general).

Así las cosas, podemos aseverar que todo conocimiento humano es factible y posible mediante el manejo de casos concretos. Por ello, todas las disciplinas han utilizado el manejo práctico de casos con el fin de penetrar y ampliar el conocimiento de sus leyes generales, y así obtener más y mejores resultados en el aprendizaje.

De ahí que las universidades de nuestro país deban de adecuar su currículo a las necesidades empresariales mediante el manejo de casos concretos en la enseñanza, lo cual lleve a la práctica del estudiante antes de ingresar al mercado laboral. Sin embargo, es preciso advertir que mientras en las ciencias naturales la premisa mayor y menor son exactas desde un principio, en los sistemas industriales técnicos la situación es muy diferente, por cuanto, dado su carácter práctico y de ciencias exactas o no - según la rama industrial de cada empresa-, cada vez que se trate de subsumir el sistema de producción a las necesidades sociales dentro de la norma general de producción, habrá nuevos aspectos que no habían sido comprendidos anteriormente, así como nuevas formas de producir y nuevas formas de productos que hagan más fácil la vida diaria.

Es así que se plantea la necesidad de generar en las universidades una conciencia con respecto a su rol y su impacto en las comunidades en las que se encuentran, a partir de la extrapolación del concepto más conocido de responsabilidad social empresarial (Brodsky, 2017, p. 15). 
En la investigación educativa se realizan estudios con la intención de lograr una mejor comprensión del proceso enseñanza-aprendizaje, así como de las condiciones en las que puede llevarse a cabo con mayor eficacia. Esto en razón a que por medio de ese proceso se pretende descubrir principios, desarrollar procedimientos aplicables en el terreno de la educación, etc. Los fenómenos que suelen ser estudiados en la investigación educativa están vinculados con la naturaleza, la eficacia y la condición del aprendizaje, aunque también se abordan temas relacionados con diferentes situaciones educacionales (Bayardo, 1995, p. 29), cuestiones que de igual manera deben ser aplicados por el estudiante cuando pasa a ser profesional (en su trabajo), esto es, buscar las condiciones para desarrollar procedimientos aplicables a la producción, sea cualquiera que sea. De ahí que el método de investigación a enseñar en las universidades sea primordial, pues será la base de aplicación para el futuro profesional en su nuevo empleo.

La aplicación de métodos y técnicas prácticas y operativas debe priviligiarse en las universidades, ya que no debe de ser una educación de oyentes, y menos aún de dictados, sino una educación de prácticas, de aplicación de tecnologías; estamos en el siglo XXI, en el cual la digitaliización e informática son aplicables en todos los terrenos y ciencias.

Etimológicamente, el término método proviene del vocablo griego meta, "hacia", y odos, "camino": "camino a seguir" (Martínez, 2000, p. 27). Por tanto, es importante no solo la enseñanza de métodos, sino su manejo y aplicación, ya que estos serán los que aplicará el futuro profesional con la finalidad de obtener mejores productos a más bajos costos.

El método enseña a encontrar las grandes hipótesis, así como las ideas nuevas y fecundas. Tiene reglas para conducir a los grandes descubrimientos científicos y engendrar las obras maestras (2000), por lo cual afirmamos que la enseñanza sobre cómo aplicar métodos científicos en las universidades debe ser primordial.

Así, podemos afirmar que el objetivo de toda ciencia es comprender y explicar las cosas, captar la relación necesaria que une una cosa a su causa o a su principio, a fin de resolver un problema al identificar la causa que lo genera.

La educación científica debe estar dirigida a fomentar la construcción de conocimientos por parte de los alumnos, en lugar de repetir sistemas de conocimientos ya elaborados; esto dará margen a generar emprendedores, no mano de obra.

Hoy las universidades son centros donde la sociedad prepara no solo la transmisión del conocimiento, sino la innovación, uno de los pilares del cambio para mejorar la calidad educativa (Villanueva, 2015, p. 21).

Esta transmisión del conocimiento se realiza a través de la investigación, mediante procesos de enseñanza-aprendizaje en los que el método del procedimiento es necesario para lograr la innovación, no solo en la educación, sino en patentar invenciones que posteriormente generarán nuevas empresas, en las cuales, seguramente, el estudiante pasara a ser emprendedor.

Entre las características que se deben desarrollar en el estudiante se encuentran una constante predisposición para aprender, la capacidad de enfrentarse a situaciones complejas y cambiantes, de resolver problemas y tomar decisiones, además de ser ciudadanos críticos y responsables (Sancho et al., 2007, p. 418).

La transformación de las demandas sociales, económicas y culturales planteadas a nivel local y global, es también una herramienta clave para lograr con éxito la inserción de los profesionales en el mercado laboral (Alcaraz, 2009, p. 13), ya que son, en este sentido, generadores de empleo y no de empleados.

Por lo tanto, si las empresas emplean métodos y procesos de producción, se debe enseñar al estudiante a aplicar los procesos de producción. Toda sociedad mercantil tiende a vender sus productos (es parte de su origen), esto es, realizar ventas mercantiles e invadir el mercado con sus productos, de ahí que será necesario preparar a los estudiantes en el área de mercadotecnia, a fin de establecer mejores procesos de venta de productos. Asimismo, al estudiante de otras ramas científicas que serán parte de la empresa, con el fin lograr su objetivo social.

La sociedad industrial ha sido superada por la hegemonía de la sociedad del conocimiento, la cual ha incidido en el cambio y la generación de reformas a nivel social y político. La educación, a lo largo de la vida, favorece el surgimiento de nuevos perfiles sociales y fortalece la competitividad de la economía, mejora la empleabilidad y la adaptabilidad de la fuerza de trabajo y representa un sustento importante de las políticas de cohesión e integración social, así como del crecimiento económico del mercado (Villanueva, 2015, p. 36). 
Este crecimiento económico del mercado implica el desarrollo de ciencias tales como el derecho y, en especial, del derecho mercantil, puesto que las ventas que realiza una empresa llevarán implícito el comercio y la ciencia que lo regula. Por tanto, resulta trascendente que los futuros abogados conozcan la materia mercantil con su procedimiento, pues será necesaria su aplicación en la actividad empresarial.

\section{La empresa en la ciencia y en las universidades}

Las empresas e industrias mexicanas deben tener mayor contacto con las universidades a efecto de que comuniquen sus necesidades técnicas y científicas para la creación y desarrollo de las carreras y cátedras.

La empresa mexicana debe integrar al nuevo profesional con una visión clara de mejorar la calidad y su producción; de no hacerlo así, estaría condenada a la desaparición. Por su parte, el profesional egresado de una universidad llega a la empresa con una visión genérica de la ciencia, con el conjunto de conocimientos listos para ser aplicados, así como con teorías, principios y modelos que generen soluciones a los problemas empresariales. De igual forma lo hace un contador, un abogado, un ingeniero, un economista, etc., con el afán e ímpetu de aplicar lo aprendido, bajo una idea clara de estructurar mejor los sistemas o políticas de producción, de manera que esto optimice los resultados que hasta ese momento tiene su empleador. Sin embargo, de nada sirve el ímpetu si no ha existido la práctica del conocimiento adquirido en las universidades.

Sin una idea exacta de lo que se busca y del camino posiblemente eficaz, ninguna empresa puede tener éxito. Razón por la cual debe establecer las condiciones necesarias para la investigación, así como proporcionar instrumentos de trabajo al científico para generar ciencia, lo que redunde en descubrimientos que sirvan al empresariado mexicano a competir a nivel mundial y generen mejores ingresos, ya que habrá generado nuevos procesos de producción -o nuevas tecnologías-, con mejores resultados. Así, el empresario podrá vender, enseñar o invertir sus procesos de producción, con su tecnología aplicada, como, por ejemplo, en el caso de las máquinas procesadoras de tortillas fabricadas en México que se venden al extranjero.
Por lo tanto, las empresas deben inmiscuirse en la generación de conocimientos y en la práctica profesional de los futuros profesionales, e ir de la mano con las universidades.

Resulta entonces prioritario coordinar acciones entre todos los actores nacionales (gobierno, sector privado, organizaciones civiles, universidades e instituciones educativas y cámaras empresariales, entre otros), con el fin de compartir conocimiento e información sobre la educación superior e identificar las prioridades para el diseño, no solo del currículo, sino también de las carreras universitarias, y así responder al desarrollo que se requiere (Rodríguez et al., 2015, p.17).

El desarrollo del mundo científico y tecnológico cambia con rapidez, lo que obliga a las instituciones educativas y a las empresas a adaptarse a las nuevas realidades, a fin de competir en mercados globales (2015).

La interacción del triángulo gobierno-empresa-universidad, asegura un desarrollo científico tecnológico que tiende a la innovación en el sector educativo y empresarial, puesto que incentiva la investigación, la creatividad e innovación, ofreciendo nuevas alternativas de bienes y servicios, $\mathrm{y}$ fomenta la competitividad.

\section{De la privatización a la mercantilización de la educación superior}

En la educación universitaria se llega hoy al extremo de una oferta educativa particular sin freno alguno por parte de las autoridades educativas, lo cual lleva a tener por encima de los intereses propios de la educación superior el interés del capital, un pensamiento económico de competencia educativa sin calidad alguna. Hoy, las universidades particulares ofertan en el mercado educativo un sinnúmero de facilidades para concluir una carrera universitaria, en mucho menor tiempo del suficiente para adquirir los conocimientos mínimos profesionales. Esto lleva al extremo de sacar al mercado laboral cantidad y no calidad, en tiempos por demás insuficientes para la preparación de un estudiante en profesional.

Es necesario tener cuidado en la creación de nuevas universidades que ven solo el aspecto económico y no el científico, de manera que egresan profesionales de calidad dudosa, quienes 
engrosan el mercado de desempleo. Ante esta realidad, corresponde a las autoridades educativas la certificación constante de la calidad educativa.

Se requiere una educación que priorice la enseñanza del conocimiento aplicado a las necesidades sociales y empresariales, la cual incentivará el desarrollo económico del país, y no una educación en la que tenga prioridad solo el aspecto económico de los dueños de las instituciones privadas educativas.

\section{La socialización de la educación}

El título de este apartado no es una propuesta política, ni la idea es politizar la educación universitaria. Sin embargo, se debe manejar, a fin de que el alumno entienda cómo la cooperación en los sistemas de enseñanza debe aplicarse de igual forma que en las empresas e industrias en las que que trabaje como profesional; esto es, no actuar por su cuenta en cualquier tema o proceso de investigación o de producción, sino en colaboración.

El desarrollo de un pueblo no depende solo del esfuerzo individual, sino de las acciones de todos los individuos capaces que unen sus esfuerzos. El logro de la habilidad para desarrollar el trabajo colectivo y cooperativo es resultado del aprendizaje inteligente del hombre (Moncayo, 1987, p. 21).

Por tanto, el catedrático debe de dar énfasis a los trabajos en equipo, con la idea no solo de la enseñanza colectiva, sino también con el afán de buscar la cooperación en la investigación, de modo que el cooperativismo lo aplique el futuro profesional en los sistemas industriales y empresariales, y el sentimiento de cooperación sirva para generar una mejor producción, tanto en calidad, como en cantidad.

Se considera que la educación es funcional cuando se forma al educando para que aprenda comportamientos que le permitan resolver los problemas cotidianos (1987, p. 23). Trabajar en equipo no es solo un medio que lleve al dominio de los asuntos del programa escolar, sino un fin: una práctica para la adquisición de hábitos, habilidades y actitudes sociales (1987), con la idea de maximizar la enseñanza, en la que se tienda a establecer para el futuro profesional la concepción de cómo en el sistema productivo se debe buscar la eficiencia y, por ende, la maximización de la producción. Todo lo anterior con miras a producir la universidad como una terminal de profesionales que cumplan con las necesidades de las empresas mexicanas.

Enseñar al estudiante a conocer y aprender a hacer deben de ir de la mano, ya que es parte esencial de la formación profesional; el aprendizaje debe ser un proceso de constante revisión y de construcción de esquemas de conocimiento. Sobre los contenidos escolares, enseñar al alumno a poner en práctica sus conocimientos y, al mismo tiempo, adaptar la enseñanza al futuro mercado laboral, cuya evolución no es previsible; prueba de esto se cuando hoy enfrentamos una crisis financiera que repercute en la producción de las empresas, las cuales requieren de menor mano de obra.

La enseñanza tiene que ayudar a establecer vínculos sustantivos y no arbitrarios entre los nuevos contenidos y los conocimientos previos. La situación de enseñanza-aprendizaje también puede considerarse como un proceso encaminado a superar retos, retos que pueden ser abordados por el académico (Zabala, 1995, pp. 36-46).

El proceso tecnológico se ha modificado; pasó de los procesos de producción y las tareas puramente físicas, a tareas de producción más intelectuales. Los empleadores piden un conjunto de competencias específicas a cada persona, lo que confirma la calificación propiamente dicha, adquirida mediante la formación técnica y profesional, el comportamiento social, la aptitud para trabajar en equipo, la capacidad de iniciativa y la de asumir riesgos.

Si a estas nuevas exigencias se añade la de un empeño personal del estudiante - lo cual debe fomentarse en las aulas-, combinado con los conocimientos teóricos y prácticos, el vínculo que se tendrá con respecto a la universidad-empresa cobrará mayor relevancia, al establecer vasos comunicantes de trabajo entre las necesidades empresariales y el currículo de las carreras universitarias.

Hoy, las universidades deben generar profesionales emprendedores, empleadores, no mano de obra o buscadores de empleo. Para esto deben existir programas universitarios que sean incubadoras de empresas y propicien procesos integrales de vinculación con los sectores productivos. La universidad no solo se debe constreñir a educar y preparar en las aulas, debe también crear proyectos emprendedores que sean el inicio de una futura empresa, en la que los alumnos inicien sus prácticas profesionales y generen información, estudios y diagnósticos sobre los diferente sectores 
económicos que sean de utilidad para la solución de problemas, la formulación de estrategias y la creación de políticas productivas que mejoren la competitividad.

Así como se ha planteado que las universidades deben enseñar a los alumnos a trabajar en equipo, de igual manera se debe establecer una relación estrecha con las empresas con el propósito de crear proyectos innovadores, tanto de productos, como de servicios. En consecuencia, los programas educativos tienen que dar pautas a proyectos comunes entre la universidad y la empresa.

Trabajar en equipo no solo deberá darse en las aulas; se debe hacer equipo con las empresas, lo que en mercadotecnia se denomina como "alianzas estratégicas", en busca de la mejora continua de la calidad.

Del mismo modo, las universidades deben asumir su rol social, pues ellas son el escalón superior del conocimiento y de la formación técnica en el campo de un saber determinado, lo que constituye un desarrollo sustentable (Brodsky, 2017, p. 22).

\section{La socialización de las empresas}

La empresa mexicana debe de estar en contacto no solo con la universidad, sino con la propia sociedad. Esto con el fin de identificar las necesidades que requiere satisfacer, lo cual la conduce a un conocimiento singular de la realidad social, bajo la idea de una producción empresarial que tienda a crear satisfactores sociales. En otras palabras, a fin de que la empresa o industria mexicana se encamine a realizar los productos que la sociedad requiere, por ejemplo: ¿Cuántas empresas mexicanas fabrican navajas de rasurar, tubos de pasta dental, contenedores, etc.?, es decir, ¿por qué importar estos productos, cuando se pueden fabricar en México, lo que significaría la creación de empleos e ingresos económicos? Bien sea en la producción de pequeños productos, o bien grandes, esta requiere de igual manera que los estudiantes tengan pleno conocimiento de las necesidades sociales, el cual se adquiere en las aulas universitarias.

El conocimiento pleno de la realidad social por parte de las empresas y la producción de los satisfactores que requiere producirá progreso, de ahí la importancia de su estudio en las organizaciones cuyo rol social, entre otros, es la generación de empleo y el progreso mismo, generado a través de descubrimientos, invenciones, transformaciones de bienes y del aumento de producción. Recordemos que el factor social de las empresas es trascendental, no solo en el papel que juegan dentro de la misma, sino también en las funciones que desempeñan; no se podría concebir una sociedad sin empresas, así como tampoco una empresa sin sociedad.

Hoy es aceptada la concepción según la cual, además de producir bienes y servicios, las empresas deben cumplir ciertos objetivos sociales. Hablar de una empresa socialmente responsable no solo implica el cumplimiento de la ley, o de las normas sociales; una empresa produce estabilidad social e ingresos al Estado. La empresa del siglo XXI debe trabajar mancomunadamente con las universidades en proyectos motivadores e innovadores, que permitan crear nuevas expectativas al futuro profesional.

Tener un pleno conocimiento de las características socioeconómicas del lugar en donde se encuentra la empresa, así como de a quién va dirigido el producto o servicio, servirá para conocer las demandas básicas de la sociedad que es, en última instancia, la consumidora del bien o servicio ofrecido.

En el mundo entero las empresas privadas se caracterizaron por perseguir, como objetivo fundamental y muchas veces excluyente, el lucro económico y la optimización de sus beneficios, y olvidaron la importancia que sus actos de responsabilidad social podían tener (Brodsky, 2017, p. 21).

\section{Conclusiones}

El Estado debe dar impulso a la educación y la investigación, con el fin de abordar los problemas sociales de manera sistemática y, sobre todo, ofrecer alternativas de soluciones pertinentes $\mathrm{y}$ viables que tiendan a resolver las necesidades sociales. Esto por medio de nuevos conocimientos que permitan reducir costos de producción y tener mayor competitividad en los mercados globalizados, lo cual implica una estrecha vinculación en la denominada "triple hélice": gobierno, universidad y empresa.

Asimismo, las empresas deben reclutar al personal mediante el anuncio abierto y con base en el mérito, al crear un sistema fuerte de ejecución en la gerencia de funcionamiento que deba 
ser vinculatorio con las universidades, y así tomar las medidas que mejoren prácticas de enseñanza y garanticen la calidad en la inversión y en la formación de capital humano. Esto a partir de un modo de educación que se adapte a las necesidades de la empresa, de manera que se satisfaga la adquisición de conocimiento que el desarrollo del país requiere para una economía global competitiva.

Las universidades de México deben adecuar su currículo de materias a las necesidades empresariales mediante el manejo de casos concretos en la enseñanza, lo que lleve a la práctica del estudiante antes de ingresar al mercado laboral, en busca de nuevas y mejores formas de producir, así como nuevos productos que hagan más fácil la vida diaria.

Por otra parte, se debe privilegiar en las universidades la aplicación de métodos y técnicas prácticas y operativas. No debe ofrecer una educación en la que el profesor sea quien solo exponga, y menos aún dicte, sino una educación de prácticas, de aplicación de tecnologías, de interacción con el alumno. Estamos en el siglo XXI, la digitalización e informática son aplicables en todos los terrenos y ciencias en busca de la educación científica, la cual debe dirigirse a fomentar la construcción de conocimientos por parte de los alumnos, en lugar de repetir sistemas de conocimientos ya elaborados; es decir, se debe fomentar la innovación, lo cual dará margen a generar emprendedores, y no solo mano de obra.

Las empresas e industrias mexicanas deben de tener mayor contacto con las universidades, a efecto de que comuniquen sus necesidades técnicas y científicas para la creación y desarrollo de las carreras y cátedras que requieren. En este sentido, la empresa mexicana debe integrar al nuevo profesional con una visión clara de mejorar la calidad y su producción, con el propósito ser más competitiva. De no hacerlo así, estará condenada a la desaparición.

La empresa debe invertir en investigación y proporcionar instrumentos de trabajo al científico para generar ciencia, lo que redunde en descubrimientos que sirvan al empresariado mexicano a competir a nivel mundial y genere mejores ingresos. Así, se generarán nuevos procesos de producción -o nuevas tecnologías- con mejores resultados, de manera que las empresas puedan vender, enseñar o invertir sus procesos de producción con su tecnología aplicada.

El catedrático debe hacer énfasis en los trabajos en equipo, con la idea no solo de la enseñanza colectiva, sino también con el afán de buscar la cooperación en la investigación. Esto con el fin de que el el futuro profesional aplique el cooperativismo en los sistemas industriales y empresariales, y el sentimiento de cooperación sirva para generar una mejor producción, tanto en calidad, como en cantidad. Enseñar al estudiante a conocer y a aprender a hacer deben de ir de la mano, es parte esencial de la formación profesional. Asimismo, enseñar al alumno a poner en práctica sus conocimientos y, al mismo tiempo, a adaptar la enseñanza al futuro mercado laboral.

Hoy en día, las universidades deben generar profesionales emprendedores, empleadores, no mano de obra o buscadores de empleo, para lo cual deben existir programas universitarios que sean incubadoras de empresas. La universidad no solo debe constreñirse a educar y preparar en las aulas, debe también crear proyectos emprendedores que sean el inicio de una futura empresa, en la que los alumnos inicien sus prácticas profesionales y así, al momento de egresar, cuenten con la teoría y la práctica que requieren.

El conocimiento pleno de la realidad social por parte de las empresas, así como la producción de los satisfactores que requiere la sociedad producirá progreso. De ahí la importancia del estudio de la sociedad por parte de las empresas, cuyo rol social, entre otros, es la generación de empleo y el progreso por medio de descubrimientos, invenciones, transformaciones de bienes y el aumento de la producción. Recordemos que el factor social de las empresas es trascendental, no solo en el papel que juegan dentro de esta, sino en las funciones que desempeñan. No se podría concebir una sociedad sin empresas, así como tampoco una empresa sin sociedad.

\section{REFERENCIAS}

Alcaraz, C., y Ordóñez, J. (1995). Acontecimientos que han configurado el espacio europeo de educación superior. Recorrido histórico. En A. González, \& Y. Checa (Coord.), Posibilidades, experiencias y retos en el espacio europeo de educación superior. España: Universidad de Granada, Editorial Qualitas.

Bayardo, M. G. (1995). Introducción a la metodología de la investigación educativa. México: Editorial Plaza y Valdez.

Brodsky, J. (2017). La educación universitaria y la élite del conocimiento: hacia un ejercicio de la responsabilidad social universitaria. Academia Revista sobre Enseñanza del Derecho, 15(29). 
Chang, H. G. (2010). El modelo de la triple hélice como un medio para la vinculación entre la universidad y empresa. Revista Nacional de Administración, 1.

Enciclopedia Jurídica Mexicana (Tomo V). (2012). México: Editorial Porrúa.

Gonzáles de la Fe, T. (2009). El modelo de triple hélice de relaciones universidad industria y gobierno, un análisis crítico. Revista Arbor. Ciencia, Pensamiento Cultura, 185(738).

Larrauri, R. (enero del 2006). La educación jurídica, como campo de investigación desde una conceptualización epistemológica. Revista Universitas, 3.

Martínez, M. (2000). Fundamentos teóricos para el proceso del diseño de un protocolo de investigación. México: Editorial Plaza y Valdez.

Moncayo, L. (1987). Como trabajar en equipo. México: Editorial Hexágono.
Rodríguez, M., Acuña, A., Rojas, R., \& Lobato, A. (2015). Vinculación universidad-empresa-Estado en Nicaragua. Revista Orbis, 11(31).

Sancho, J. M., Hernández, F., Jörg, M., Larraín, V., Casablancas, S., \& Bosco, A. (2007). Innovación educativa en Europa. Aprender de la colaboración. En Investigar el cambio curricular en el espacio Europeo de educación superior. España: Universidad de Alicante, Editorial Marfil.

Villanueva, R. (2015). Análisis de dos discursos sobre la educación superior en "La transmisión del conocimiento". En R. Contreras (Coord.). México: Editorial Universidad de Guadalajara.

Zabala, A. (1995). La práctica educativa. Cómo enseñar. España: Editorial Graó. 